\title{
Capacidades resilientes en docentes ante casos de violencia intrafamiliar
}

\author{
Resilient capacities of teachers against cases of intra-family violence
}

\author{
Amalia Daniela Oviedo ${ }^{a}$ ORCID: 0000-0002-0296-743X \\ Katherine Tarazona Meza ${ }^{\mathrm{b}}$ ORCID: 0000-0002-5126-3439 \\ Angie Lorena Ramírez ${ }^{c}$ ORCID: 0000-0002-9385-6478
}

Recibido: 11/12/2019 • Aprobado: 24/01/2020

Cómo citar: Oviedo, A. D., Tarazona Meza, K., \& Lorena Ramírez, A. (2020). Capacidades resilientes en docentes ante casos de violencia intrafamiliar. Ciencia y Educación, 4(1), 21-31. Doi: https://doi.org/10.22206/cyed.2020.v4i1.pp21-31

\section{Resumen}

La investigación tuvo como objetivo identificar las capacidades resilientes que poseen los docentes de la Escuela General Básica Fiscal 24 de Mayo N², ubicada en la ciudad Portoviejo, Ecuador, en el afrontamiento de casos de violencia intrafamiliar. La metodología utilizada fue de tipo cuali-cuantitativo, a través de métodos descriptivos y deductivos. Los instrumentos fueron el test SV-RES (Saavedra \& Villalta, 2008) -para medir la resiliencia-, la encuesta y la entrevista semiestructurada. La investigación fue realizada con 47 docentes, cantidad que constituye el $100 \%$ de la población, durante los meses de septiembre y octubre del año 2019. Los resultados arrojaron que, en general, los docentes poseen capacidades resilientes promedio y alta, y que en el afrontamiento de estos casos cuentan con rutas y protocolos a seguir para la detección y derivación de estos; sin embargo, utilizan también sus habilidades personales para saber sobrellevar los casos de violencia intrafamiliar.

Palabras clave: competencias del docente; violencia doméstica; habilidad; efectos psicológicos; bienestar social.

\begin{abstract}
This investigation aims to identify resilient capacities of teachers at Escuela General Básica Fiscal 24 de Mayo No. 2, Portoviejo, Ecuador, at coping cases of domestic violence. A qualitative-quantitative methodology was used, as well as descriptive/deductive methods; SV-RES test (Saavedra \& Villalta, 2008) was used to measure resilience, a survey and a semi-structured interview. The research was conducted with 47 teachers who represent 100\% of the population, during September and October of 2019. Results show that in general teachers have medium and high resilient capacities and for coping these cases they have routes and protocols for detection and derivation of them. It was found that teachers also use their personal skills in order to cope cases of domestic violence.
\end{abstract}

Keywords: Teacher competencies; domestic violence; ability; psychological effects; social welfare.

\footnotetext{
a Universidad Técnica de Manabí, Ecuador. Correo-e: aoviedo@utm.edu.ec

b Universidad Técnica de Manabí, Ecuador. Correo-e: atarazona@utm.edu.ec

c Universidad Técnica de Manabí, Ecuador. Correo-e: lore910812@hotmail.com
} 


\section{Introducción}

En el rol docente existen diferentes exigencias profesionales, pues es necesario garantizar el aprendizaje en la diversidad de cada uno de sus estudiantes. El entorno familiar es uno de los elementos que influye en este proceso. En esta investigación se consideró la violencia intrafamiliar (VIF) como un elemento que puede incidir negativamente en el estudiante; según las estadísticas del Instituto Nacional de Estadísticas y Censos (INEC) esta es una realidad para un alto porcentaje de niños, nińas y adolescentes (NNA) ecuatorianos.

Uno de los requerimientos a los docentes es el trabajo con estudiantes que viven en un entorno de VIF. Si bien es posible asegurar que el Ministerio de Educación (MINEDUC) y el estado ecuatoriano cuentan con protocolos de actuación dentro de las unidades educativas, estos no consideran el desgaste personal que pueden tener los docentes al trabajar cotidianamente con esta población.

$\mathrm{Al}$ ser el docente una figura importante en la vida de los estudiantes, es necesario reconocerlo más allá de su ejercicio profesional y más que como un cumplidor de protocolos, sino como un ser biopsicosocial, que tiene diferentes capacidades y características personales, siendo la resiliencia una de ellas. Esto incide en la relación que genera con sus estudiantes, no solo en lo referente a lo académico, sino también como un soporte para el afrontamiento y la superación de la adversidad vivida, con lo cual -indirectamente- el bienestar psicológico del docente se ve influenciado por el trabajo que realiza.

En el Ecuador, los NNA constituyen un grupo de atención prioritaria y se encuentran afectados por diferentes tipos de violencia, destacando la VIF. Con relación a los diferentes tipos de violencia y su incidencia en la población en general, los reportes del INEC (2018) destacan las siguientes provincias:

Violencia psicológica en las provincias de Morona Santiago (58,6\%), Pastaza (53,9\%) y Tungurahua $(53,7 \%)$. Violencia física en las provincias de Pastaza (44,9\%), Tungurahua $(44,7 \%)$ y Morona Santiago (44,6\%). Violencia sexual en las provincias de Morona Santiago (22,3\%), Zamora Chinchipe (19,7\%) y Cañar
(18,3\%); mientras que, en Manabí, violencia psicológica $(32,0 \%)$, violencia física $(24,9 \%)$ y violencia sexual (8,7\%) (p. 360).

En el año 2012, mediante el convenio de cooperación interinstitucional MINEDUC (2017), se establece que el Consejo de la Judicatura y la Fiscalía General del Estado "garantice espacios libres de violencia mediante la prevención, atención, protección, investigación y restitución inmediata de los derechos vulnerados evitando la re-victimización de los (NNA) que han sido víctimas de violencia” (p. 9).

Los índices establecidos por el INEC sugieren que dentro de los hogares de Ecuador la violencia puede ser un elemento común, con lo cual los docentes resultan ser una piedra angular de identificación en este sentido. De acuerdo a los "Protocolos de actuación frente a situaciones de violencia detectadas o cometidas en el sistema educativo" (MINEDUC, 2017), los docentes, las autoridades, el personal administrativo y los padres de familia, así como los actores de la sociedad civil deben trabajar en conjunto para la erradicación de la violencia hacia los menores de edad.

Es necesario considerar el nivel de aplicación y el impacto de las políticas públicas que buscan la protección de los NNA. Restrepo \& García (2017) hacen referencia a diversos estudios que resaltan que en Perú, Brasil y Chile los agentes educativos bien capacitados tienen una mayor eficacia al momento de identificar abusos sexuales en las unidades educativas. Los autores reconocen, además, que debido a las desarticulaciones que existen entre las instituciones y los programas las intervenciones se ejecutan de una manera fragmentada (p. 32), evidenciando esto que la participación de varias instituciones genera dificultades al momento de la implementación de acciones de protección.

Es necesario establecer la relación que tiene la efectividad de los protocolos de actuación y el papel activo de la escuela en el abordaje de los casos de niños, niñas y adolescentes víctimas de violencia intrafamiliar (NNAVIF). Wang et al. (1994, citado por Acevedo \& Mondragón, 2005) afirman que "los maestros pueden tener un rol muy importante en reducir el estrés, dando el apoyo positivo que tanto necesitan los nińos en condiciones adversas" (p. 27). 
En un estudio realizado en Argentina por Forján, R. \& Morelatto G. (2018) sobre la resiliencia en docentes, en el que utilizaron como instrumento la escala de resiliencia docente ER-D de Guerra, (2013, citado por Forján, R. et al, 2008), se evidenció que los docentes de contextos vulnerables presentan valores elevados en los rangos alto y medio de resiliencia en la participación significativa (p. 286). Por otro lado, Díaz \& Barra (2017) reflejaron en su investigación que el nivel reportado por los docentes para la variable resiliencia es bastante alto; estos investigadores utilizaron como instrumento la escala de resiliencia SV-RES.

Diferentes estudios realizados confirman que la resiliencia disminuye la vulnerabilidad al burnout. Aunado a esto, una de las tendencias clave de la resiliencia es la capacidad de afrontamiento a los estresores (Vicente \& Gabari, 2019, p. 108); por tanto, debe ser una característica fundamental para los docentes al mediar los conocimientos con la parte psicoemocional de los NNAVIF.

Debido al nivel de incidencia de casos de VIF en el Ecuador y a las consecuencias que ejercen en la vida de los NNA, considerando al docente como un ser biopsicosocial, este estudio busca identificar las capacidades resilientes que poseen los docentes de la Escuela General Básica Fiscal 24 de mayo No. 2, ubicada en la ciudad Portoviejo, Ecuador, así como evidenciar el tipo de estrategias de afrontamiento adicionales a los protocolos establecidos por el MINEDUC y el gobierno.

\section{Marco teórico}

En el Ecuador, la Ley Contra la Violencia a la Mujer y la Familia, en el artículo 2 (2018), define la VIF como "toda acción u omisión que consista en el maltrato físico, psicológico o sexual, ejecutado por un miembro de la familia en contra de la mujer o demás integrantes del núcleo familiar". En cuanto a la tipología de la violencia, el artículo 4 refiere que existe:

Violencia física: todo acto de fuerza que cause daño, dolor o sufrimiento físico en las personas agredidas, cualquiera que sea el medio empleado y sus consecuencias.
Violencia psicológica: constituye toda acción u omisión que cause daño, dolor, perturbación emocional, alteración psicológica o disminución de la autoestima de la mujer o familiar agredido.

Violencia sexual: se considera violencia sexual la imposición en el ejercicio de la sexualidad de una persona a la que se le obligue a tener relaciones o prácticas sexuales con el agresor o con terceros, mediante el uso de fuerza física, intimidación, amenazas o cualquier otro medio coercitivo (INEC, 2018, p. 360).

Para el abordaje de la VIF es necesario considerar que esta tiende a perpetuarse como parte de la organización familiar, determina la creación de vínculos afectivos de los sujetos y ocurre en el espacio familiar, por lo cual, al ser un espacio privado, su detección resulta aún más compleja (Muñoz \& Sotelo, 2005, p. 112).

Si las instituciones educativas no se involucran en la detección de casos de NNAVIF, se dificulta garantizar derechos a esta población. El Plan Nacional para la erradicación de la violencia de género hacia la niñez, adolescencia y mujeres (2009) considera que las unidades educativas son el ente imprescindible para la identificación, la prevención y la disminución de estos casos. Los centros educativos tienen un compromiso con el bienestar de sus estudiantes, por lo que deben brindar atención y respaldo a los NNA víctimas de situaciones de desprotección, notificando a las instancias pertinentes para las actuaciones de protección oportunas (Muñoz \& Sotelo 2005, p. 108).

La ruta de protocolos en el Ecuador frente a situaciones de VIF es conocida por los docentes, pues son capacitados y cuentan con el manual físico en las unidades educativas. Según el MINEDUC (2017), la existencia de este manual permite una perspectiva teórica homogénea sobre los tipos de violencia y facilita la detección de casos y su derivación. Es evidente que existe desarticulación en el seguimiento y la continuidad del proceso llevado a cabo en los casos; por ende, los docentes se ven en la necesidad de hacer uso de sus capacidades personales, como puede ser su resiliencia.

El concepto de resiliencia se ha transformado con el paso de los años, hasta llegar a concebirse desde la 
psicología como la capacidad de las personas para sobrevivir en condiciones adversas, desarrollando conductas de afrontamiento y de superación exitosas (Saavedra, Arévalo, Gajardo, L. \& Toledo, 2010, p. 13).

Si bien no se nace resiliente, una persona puede hacerse resiliente debido a los avatares de la vida y a la manera en que supera dificultades y adversidades. The International Resilience Project, Canadá (citado por Saavedra, E. \& Villalta, M., 2008a) manifiesta que la resiliencia es "una capacidad universal que permite a una persona, grupo o comunidad prevenir, minimizar o superar los efectos perjudiciales de la adversidad" (p. 10); al ser una capacidad que se desarrolla, es posible esperar que dentro de las unidades educativas los docentes la desarrollen como estrategia para el abordaje de casos de VIF.

Saavedra (2003), citado por Saavedra, E. \& Villalta, M. (2008b), refiere que para comprender la resiliencia es necesario considerar que:

1) La respuesta resiliente es una acción orientada a metas; respuesta sustentada o vinculada a 2) una visión abordable del problema; como conducta recurrente en 3) visión de sí mismo, caracterizada por los elementos afectivos y cognitivos positivos o proactivos ante los problemas; los cuales tienen como condición histórico-estructural a 4) condiciones de base, es decir, un sistema de creencias y vínculos sociales que impregnan la memoria de seguridad básica y que de modo recursivo interpreta la acción específica y los resultados (p. 32) (ver Tabla 1).

Saavedra et al. (2008b), en el test SV-RES, proponen evidenciar 12 factores que consisten en "describir distintas modalidades de interacción del sujeto: consigo mismo, con los otros, con sus posibilidades" (p. 32) (ver Tabla 1).

Tabla 1. Clasificación de los factores resilientes de acuerdo al test SV-RES

\begin{tabular}{|c|c|c|c|c|}
\hline & $\begin{array}{c}\text { Condiciones } \\
\text { de base }\end{array}$ & $\begin{array}{c}\text { Visión de sí } \\
\text { mismo }\end{array}$ & $\begin{array}{c}\text { Visión del } \\
\text { problema }\end{array}$ & $\begin{array}{c}\text { Respuesta } \\
\text { resiliente }\end{array}$ \\
\hline $\begin{array}{c}\text { Yo } \\
\text { soy, yo } \\
\text { estoy }\end{array}$ & $\begin{array}{c}\text { F1: } \\
\text { Identidad }\end{array}$ & $\begin{array}{c}\text { F2: } \\
\text { Autonomía }\end{array}$ & $\begin{array}{c}\text { F3: } \\
\text { Satisfacción }\end{array}$ & $\begin{array}{c}\text { F4: } \\
\text { Pragmatismo }\end{array}$ \\
\hline
\end{tabular}

\begin{tabular}{|c|c|c|c|c|}
\hline & $\begin{array}{c}\text { Condiciones } \\
\text { de base }\end{array}$ & $\begin{array}{c}\text { Visión de sí } \\
\text { mismo }\end{array}$ & $\begin{array}{c}\text { Visión del } \\
\text { problema }\end{array}$ & $\begin{array}{c}\text { Respuesta } \\
\text { resiliente }\end{array}$ \\
\hline $\begin{array}{c}\text { Yo } \\
\text { tengo } \\
\ldots\end{array}$ & $\begin{array}{c}\text { F5: } \\
\text { Vínculos }\end{array}$ & $\begin{array}{c}\text { F6: } \\
\text { Redes }\end{array}$ & $\begin{array}{c}\text { F7: } \\
\text { Modelos }\end{array}$ & $\begin{array}{c}\text { F8: } \\
\text { Metas }\end{array}$ \\
\hline $\begin{array}{c}\text { Yo } \\
\text { puedo }\end{array}$ & $\begin{array}{c}\text { F9: } \\
\text { Afectividad }\end{array}$ & $\begin{array}{c}\text { F10: } \\
\text { Autoeficacia }\end{array}$ & $\begin{array}{c}\text { F11: } \\
\text { Aprendizaje }\end{array}$ & $\begin{array}{c}\text { F12: } \\
\text { Generatividad }\end{array}$ \\
\hline
\end{tabular}

Fuente: Matriz de resiliencia Saavedra, E. \& Villalta, M. (2008).

Los factores de resiliencia que proponen Saavedra et al. (2008a) son:

F1: Identidad. (Yo soy-Condiciones de base) Valores culturales que definen al sujeto, es decir, su caracterización personal.

F2: Autonomía. (Yo soy-Visión de si mismo) Vínculo que establece el sujeto consigo mismo para definir su aporte al entorno sociocultural (p. 31).

F3: Satisfacción. (Yo soy-Visión del problema) Como interpreta una situación problemática.

F4: Pragmatismo. (Yo soy-Respuesta resiliente) Interpretación de las acciones que realiza.

F5:Vinculos. (Yotengo-Condiciones base)Socialización y redes sociales de la historia personal.

F6: Redes. (Yo tengo-Visión de sí mismo) Vínculo afectivo de la persona con su entorno social cercano (p. 32).

F7: Modelos. (Yo tengo-Visión del problema) Convicción sobre el apoyo de las redes sociales cercanas para la superación de nuevas problemáticas.

F8: Metas. (Yo tengo-Respuesta resiliente) Metas y redes sociales por encima de la problemática.

F9: Afectividad. (Yo puedo-Condiciones base) Posibilidades de auto-reconocimiento y el vínculo con el entorno (p. 33).

F10: Autoeficacia. (Yo puedo-Visión de sí mismo) Posibilidades de éxito que reconoce en sí mismo ante una situación problemática.

F11: Aprendizaje. (Yo puedo-Visión del problema) Valorar una situación problemática como un aprendizaje.

F12: Generatividad. (Yo puedo-Respuesta resiliente) Posibilidad de pedir ayuda para solucionar las situaciones problemáticas (p. 34). 
He aquí la importancia de la resiliencia en los docentes para afrontar los casos de VIF, pues si estos se conocen a sí mismos, tendrán mayores capacidades para exteriorizar sus valores, creencias y conocimientos, denotando seguridad y control ante situaciones estresantes o adversas (Gómez \& Almeida 2016, p. 170). Esta no es una problemática que afecta solo al ámbito privado de la familia; el docente dentro de las unidades educativas debe sobrellevar y disminuir las posibles consecuencias de los NNAVIF: bajo rendimiento académico, conductas desafiantes, depresión, falta de control de los impulsos, delincuencia, deserción escolar, entre otras. Es decir que, si los docentes no logran proporcionar respuestas a las limitaciones familiares y sociales de los estudiantes, estas consecuencias negativas seguirán presentándose. Uriarte (2006) reconoce la importancia del acompañamiento y el seguimiento, a fin de alcanzar las problemáticas que viven los NNA (p. 8).

Por lo antes expuesto, es necesario que los docentes no solo posean los conocimientos para impartir sus clases, sino que en el proceso de enseñanza aprendizaje estos guíen hacia el desarrollo personal y fomenten estrategias que permitan a sus estudiantes enfrentar la adversidad (VIF) o las situaciones complejas. Como mencionan Forján et al. (2018), las escuelas que trabajan con poblaciones en situaciones vulnerables, que atraviesan problemáticas sociales, precisan que los docentes les hagan frente desde sus recursos personales y contextuales (p. 279). De este modo, la empatía que un docente establezca con sus estudiantes puede ser una herramienta de abordaje, así como el conocimiento del desarrollo emocional y social de sus estudiantes.

De acuerdo con Lagos \& Ossa (2010), si la capacidad de resiliencia de los docentes es alta, esta permite fortalecer en ellos su habilidad de afrontamiento al momento de educar a estudiantes que viven en ámbitos violentos (p. 43); por consiguiente, al ser estas habilidades eficaces permitirán que el docente pueda acompañar de manera apropiada los casos de NNAVIF.

Según investigaciones realizadas por Saltijeral \& Ramos (2015), debido a las exigencias que enfrenta el docente en los casos de NNAVIF, y al verse obligado a desarrollar nuevas estrategias para brindar apoyo, es posible que se produzca agotamiento, provocando estrés laboral (p. 362), lo cual influiría en la manera en que sean abordadas estas problemáticas.

Trabajar con poblaciones en condiciones de vulnerabilidad representa entonces un desgaste adicional para los docentes. Franco (2010) señala que los profesionales que tienen como objeto el trabajo con personas en sectores de situaciones de riesgo pueden sufrir burnout, debido al nivel de exigencia afectivo y emocional que requiere el abordaje. Como se ha expuesto, la exigencia al docente en los casos del NNAVIF no termina en la aplicación de protocolos. El docente no solo debe velar por la garantía de derechos de los NNA (acceso a la educación), sino que además se evalúa su forma de sobrellevar las dificultades relacionadas con los casos de VIF en el salón de clase.

\section{Metodología}

El tipo de estudio realizado es cuali-cuantitativo, de metodología descriptiva. El estudio fue realizado con el $100 \%$ de la población total, que corresponde a 47 docentes de la Escuela General Básica Fiscal 24 de mayo No. 2, de la ciudad de Portoviejo, Ecuador; de estos, 39 son mujeres (83\%) y 8 son varones (17\%); a su vez, se muestran por rangos de edad (ver Tabla 2).

Para la elaboración de este estudio se consideraron las variables de niveles de resiliencia y el tipo de afrontamiento que utilizan frente a los casos NNAVIF. Se debe resaltar que no se consideró el nivel de aplicación de los protocolos, pues se buscó evidenciar el tipo de estrategias que los docentes utilizan, complementarias a la aplicación de los protocolos, y cómo el trabajo con estos casos les afecta emocionalmente.

Asimismo, es necesario señalar que, durante el proceso de recolección de datos y análisis, la identidad de los docentes fue manejada de manera anónima y se garantizó la confidencialidad de la información recolectada. Todos los docentes que participaron en la investigación lo hicieron de manera voluntaria y con el aval de la unidad educativa. 
Tabla 2. Rangos de edad, etapa de desarrollo y número de docentes representados en la muestra

\begin{tabular}{|c|c|c|c|}
\hline $\begin{array}{c}\text { Rango } \\
\text { en años })\end{array}$ & $\begin{array}{c}\text { Etapa de } \\
\text { desarrollo }\end{array}$ & $\begin{array}{c}\text { Número } \\
\text { de } \\
\text { docentes }\end{array}$ & $\begin{array}{c}\text { Porcentaje } \\
\text { de la } \\
\text { muestra }\end{array}$ \\
\hline $20-40$ & $\begin{array}{c}\text { Adultez } \\
\text { temprana }\end{array}$ & 17 & $36 \%$ \\
\hline $41-60$ & $\begin{array}{c}\text { Adultez } \\
\text { media }\end{array}$ & 29 & $62 \%$ \\
\hline 61 y más & $\begin{array}{c}\text { Adultez } \\
\text { tardía }\end{array}$ & 1 & $2 \%$ \\
\hline Total & & 47 & $100 \%$ \\
\hline
\end{tabular}

Fuente: elaboración propia a partir de los datos recopilados en el estudio.

A fin de identificar los niveles de resiliencia en los docentes, se utilizó la Escala de resiliencia SV-RES (Saavedra \& Villalta, 2008), la cual consta de 60 ítems que permiten evaluar doce factores específicos de resiliencia (Tabla 1), cuya validez de acuerdo al Coeficiente Pearson es de $r=0,76$ y su confiabilidad según el Alfa de Cronbach es de 0,96. Para identificar los tipos de afrontamiento de los docentes con los NNAVIF se elaboró una encuesta que contó con 7 preguntas y una entrevista de 8 preguntas semiestructuradas, la cual tuvo una duración de aproximadamente 25 a 30 minutos con cada docente.

Los resultados de las entrevistas fueron analizados considerando tres unidades de análisis: 1. Estrategias personales del docente; 2 . Percepción de eficacia y limitaciones de protocolos de actuación en VIF; y 3. Afectación en la estabilidad emocional por el trabajo con NNAVIF. Posteriormente, se procedió a triangular los resultados de las entrevistas con los de las encuestas, presentando los primeros de manera también cuantitativa, según la frecuencia de respuesta.

\section{Resultados}

A continuación, se presentan los resultados obtenidos a partir de la aplicación del test SV-RES de Saavedra \& Villalta (2008), para lo cual se mostrará la cantidad de docentes por niveles de resiliencia y por rangos de edad (ver Gráfico 1), los resultados por porcentaje general de resiliencia, diferenciando por sexo (ver Tabla 3) y, finalmente, debido a que el test permite obtener resultados por factores de resiliencia, se presentarán en porcentajes (ver Tabla 4).

\section{Puntaje general de resiliencia por rangos de edad}

Gráfico 1. Nivel de resiliencia general de la población estudiada por rangos de edad

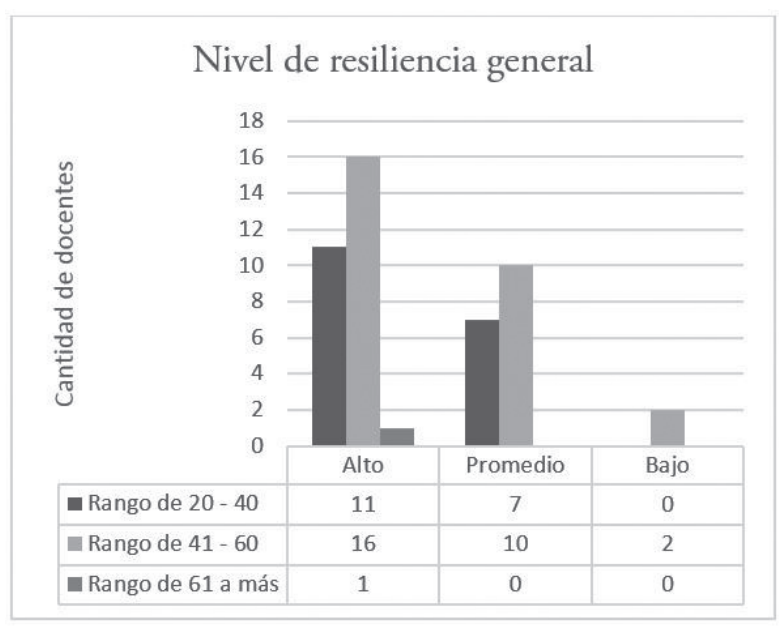

Fuente: elaboración propia a partir de los datos recopilados en el estudio.

Nota: el gráfico se realizó siguiendo los parámetros y la calificación del manual SV-RES (Saavedra \& Villalta 2008), Portoviejo, Ecuador.

La población estudiada estuvo constituida por 39 mujeres y 8 hombres. Los resultados obtenidos del nivel de resiliencia por rangos de edad (Gráfico 1) muestran que, de 18 docentes que se encuentran en el rango comprendido entre 20 y 40 años, 11 tienen resiliencia alta y 7 resiliencia promedio. En el rango de edad de 41 a 60 años existen 28 docentes, de los cuales 16 tienen resiliencia alta, 10 promedio y 2 tienen resiliencia baja. En el rango de 61 años o más solo existe un docente cuyo nivel de resiliencia es alto. 
Tabla 3. Nivel de resiliencia general en hombres y mujeres

\begin{tabular}{|c|c|c|c|c|c|}
\hline $\begin{array}{c}\text { Resiliencia } \\
\text { general }\end{array}$ & \# Hombres & $\%$ & \# Mujeres & $\%$ & $\%$ Total \\
\hline Alta & 3 & 6 & 25 & 54 & 60 \\
\hline Promedio & 4 & 8 & 13 & 28 & 36 \\
\hline Baja & 1 & 2 & 1 & 2 & 4 \\
\hline Total & 8 & & 39 & & 100 \\
\hline
\end{tabular}

Fuente: elaboración propia a partir de los datos recopilados en el estudio y realizada siguiendo los parámetros, la calificación y la clasificación del manual SV-RES (Saavedra \& Villalta, 2008).

Los resultados obtenidos, diferenciando según el sexo, arrojan que el $60 \%$ de los docentes de la escuela en cuestión tienen resiliencia alta, de los cuales el 54\% son mujeres y el $6 \%$ son hombres; el $36 \%$ tiene resiliencia promedio, siendo el $28 \%$ mujeres y el $8 \%$ hombres; y el $4 \%$ presenta resiliencia baja, representando en hombres y mujeres el $2 \%$.

Tabla 4. Nivel de factores de resiliencia por sexo

\begin{tabular}{|l|c|c|c|}
\hline $\begin{array}{l}\text { Factores de } \\
\text { resiliencia }\end{array}$ & $\begin{array}{c}\text { \% Nivel } \\
\text { alto }\end{array}$ & $\begin{array}{c}\text { \% Nivel } \\
\text { promedio }\end{array}$ & $\begin{array}{c}\% \text { Nivel } \\
\text { bajo }\end{array}$ \\
\hline F1 Identidad & 79 & 15 & 6 \\
\hline F2 Autonomía & 62 & 38 & 0 \\
\hline F3 Satisfacción & 57 & 43 & 0 \\
\hline F4 Pragmatismo & 66 & 30 & 4 \\
\hline F5 Vínculos & 53 & 45 & 2 \\
\hline F6 Redes & 66 & 32 & 2 \\
\hline F7 Modelos & 30 & 62 & 8 \\
\hline F8 Metas & 38 & 60 & 2 \\
\hline F9 Afectividad & 49 & 47 & 4 \\
\hline F10 Autoeficacia & 51 & 49 & 0 \\
\hline F11 Aprendizaje & 60 & 36 & 4 \\
\hline F12 Generatividad & 49 & 49 & 2 \\
\hline
\end{tabular}

Fuente: elaboración propia a partir de los datos recopilados en el estudio, realizada siguiendo los parámetros de calificación y clasificación del manual SV-RES (Saavedra \& Villalta, 2008), Portoviejo, Ecuador.

De acuerdo con los resultados por factores de resiliencia (ver Tabla 4) y de acuerdo a su clasificación (ver Tabla 2), se obtuvo que:
En el F1 de condiciones base de "yo soy, yo estoy", el $79 \%$ es alta; el $15 \%$ es promedio y el $6 \%$ es baja. El F2 de "visión de sí mismo", el 62\% es alta y el 38\% es promedio. El F3 de "visión del problema", el 57\% es alta y el $43 \%$ es promedio. El F4 de "respuesta resiliente", el 66\% la tiene alta; el 30\% promedio y un $4 \%$ la tiene baja.

En el F5 de condiciones base de "yo tengo": el $53 \%$ es alta; el $45 \%$ es promedio y en un $2 \%$ es baja. El F6 de "visión de sí mismo", el 66\% es alta; el 32\% es promedio y en un $2 \%$ es baja. El F7 de "visión del problema”, el $30 \%$ es alta, el $62 \%$ promedio y en un $8 \%$ es baja. El F8 de "respuesta resiliente", el 38\% la tiene alta; el $60 \%$ promedio y en un $2 \%$ es baja. Al existir un nivel bajo en "yo tengo" del F5 (Vínculos) como condición base, provoca que el F6 (Redes) "visión de sí mismo" se vea afectado y afecte, a su vez, el F7 (Modelos) de la "visión del problema"; y, finalmente, haría que la respuesta resiliente F8 (Metas) presente niveles bajos; no obstante, existe un mayor porcentaje de docentes que presentan factores en niveles altos y promedios.

En el F9 de condiciones base de "yo puedo", el 49\% la tiene alta; el $47 \%$ promedio y en un $4 \%$ es baja. El F10 de "visión de sí mismo", el 51\% la tiene alta y el 49\% promedio. El F11 de "visión del problema", en el 60\% es alta; el $36 \%$ promedio y en un $4 \%$ es baja. El F12 de "respuesta resiliente", el 49\% la tiene alta; el 49\% promedio y el 2\% la tiene baja. Al existir un nivel bajo en "yo puedo" del F9 (Afectividad) como condición base, posiblemente afecta el F11 (Aprendizaje) como visión del problema, que afecta el F12 (Generatividad) como respuesta resiliente. Sin embargo, existe un mayor porcentaje de docentes con niveles alto y promedio en estos factores.

\section{Resultados de encuesta y entrevista}

\subsection{Percepción de eficacia y limitaciones de pro- tocolos de actuación en VIF}

Los docentes refirieron que los protocolos del MINEDUC son válidos; sin embargo, consideran que muchas de las acciones llevadas a cabo no lograban 
ejecutarse ya que los casos de VIF son recurrentes y sobrepasan la capacidad de respuesta del Departamento de Consejería Estudiantil (DECE) y de otras instituciones especializadas en el trabajo con casos de violencia. Además, refieren que existe una demora al momento de manejar estos casos, incluso cuando es necesario apartar a los NNA de su núcleo familiar. Debido a los lineamientos de los protocolos establecidos, el accionar del docente se ve limitado. Señalan también que, al intentar hablar con el cuidador del niño, en ocasiones han sido maltratados verbalmente e incluso han recibido amenazas.

Gráfico 2. Resultados de la encuesta sobre la pregunta No. 4 aplicada a los docentes de la Escuela General Básica 24 de mayo No. 2, Portoviejo, Ecuador.

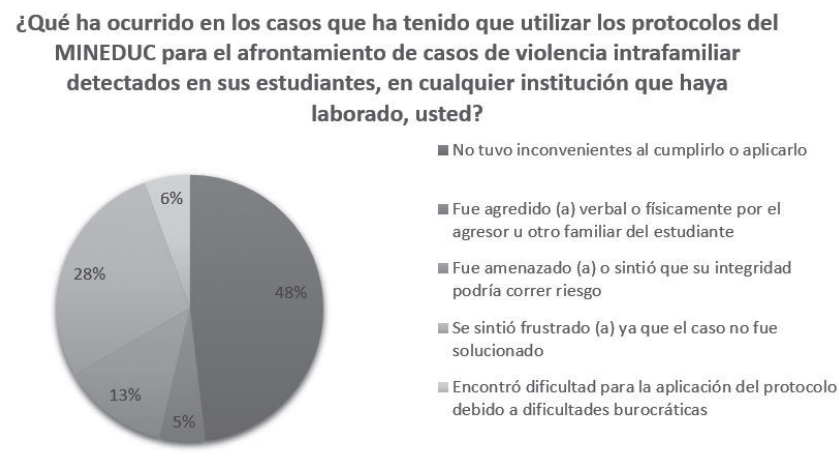

Fuente: elaboración propia a partir de los datos recopilados en el estudio.

\subsection{Estrategias personales del docente}

En esta unidad de análisis se pudo evidenciar que, a pesar de las limitaciones propias de los protocolos de actuación, los docentes buscan realizar un acercamiento directo con los casos de NNAVIF, ya sea a partir del diálogo, abrir un espacio de confianza, incluirlos dentro del grupo promoviendo iniciativas de integración, realizar un mayor acompañamiento académico, modelar su asertividad al momento de establecer límites y disciplina, establecer un acercamiento afectivo, entre otras estrategias de afrontamiento. Los docentes, además, buscan un acercamiento a los cuidadores de los NNAVIF a partir del seguimiento académico, a fin de realizar un seguimiento de los cambios o las mejoras que se puedan presentar.

A través de la encuesta se determinó que un $47 \%$ de los docentes remite directamente el caso al DECE; el $27 \%$ habla directamente con el estudiante sobre la situación de posible violencia; el 21\% utiliza estrategias curriculares para mejorar su rendimiento académico; el 3\% planteó hablar con los padres; y el 2\% señaló que es más permisivo con la conducta o las tareas del estudiante (ver Gráfico 3).

Gráfico 3. Resultados de la encuesta sobre la pregunta No. 5 aplicada a los docentes de la Escuela General Básica 24 de mayo No. 2, Portoviejo, Ecuador.

¿Qué hace usted cuando se entera o sospecha que un estudiante está siendo víctima de violencia intrafamiliar o vive en un ámbito de violencia intrafamiliar?

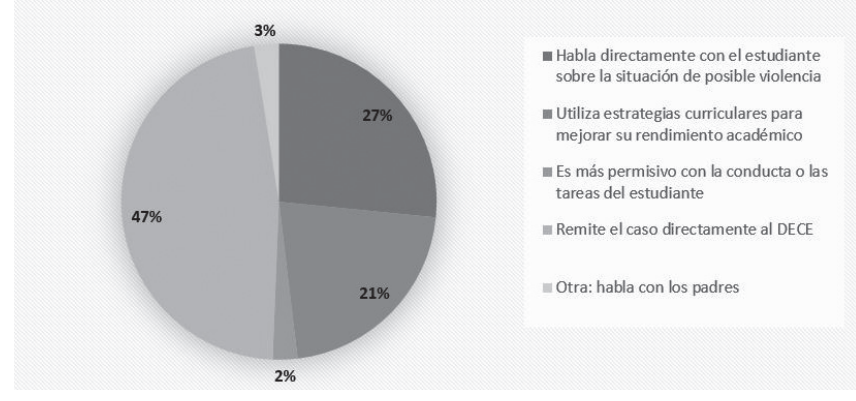

Fuente: elaboración propia a partir de los datos recopilados en el estudio.

\subsection{Afectación en la estabilidad emocional por el trabajo con NNAVIF}

Los docentes manifiestan que al trabajar con NNAVIF se ven afectados negativamente por esta realidad, refiriendo sentimientos de dolor, impotencia y poca valía, sobre todo porque consideran que, en muchos casos, no pueden cambiar la realidad que viven sus estudiantes.

Debido al tiempo que comparten y trabajan con sus estudiantes, los docentes argumentan que generan afecto hacia ellos y que es difícil ver los casos de violencia intrafamiliar solamente como un "protocolo a seguir". El 71\% de los docentes refieren que al menos algún caso de NNAVIF les ha afectado emocionalmente (ver Gráfico 4). 
Gráfico 4. Resultados de la encuesta sobre la pregunta No. 6 aplicada a los docentes de la Escuela General Básica 24 de mayo No. 2, Portoviejo, Ecuador.

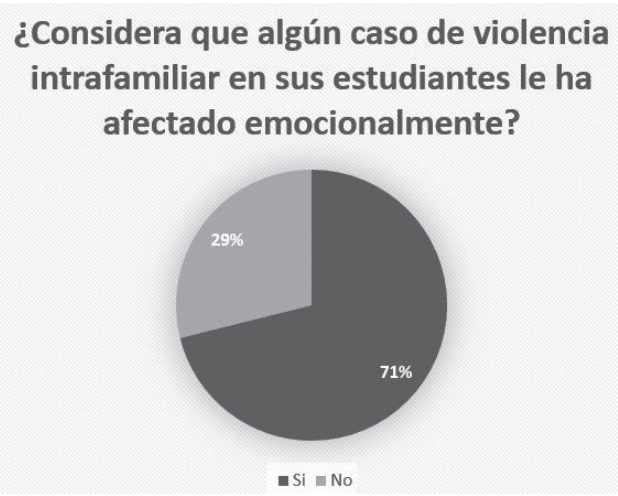

Fuente: elaboración propia a partir de los datos recopilados en el estudio.

\section{Discusión y conclusiones}

Los resultados indican que, en la mayoría de los docentes, la resiliencia se encuentra en niveles altos y promedios, así como en los factores de resiliencia de "yo tengo" (F5, F6, F7, F8) y "yo puedo" (F9, F10, F11, F12). Investigaciones previas, como las de Forján et al. (2018) y Díaz et al. (2017), han identificado que docentes que poseen resiliencia alta pueden desarrollar alternativas efectivas ante las situaciones de NNAVIF. Díaz et al. (2017) manifiestan que las condiciones personales del docente permiten determinar la manera de enfrentar los desafíos en su labor profesional.

Este estudio evidenció que el docente como profesional debe velar por el bienestar de sus estudiantes e impedir que sean vulnerados sus derechos; sin embargo, corren el riesgo de ver afectada su propia integridad en el proceso. Folco (2013) determinó que los docentes no suelen sentirse preparados para enfrentar situaciones de VIF, sino que acostumbran a estar en una situación de indefensión y amenaza. Ecuador cuenta con protocolos de actuación frente a estos casos, pero no se consideran los factores personales ni las represalias que pueden recibir ellos.

Por otra parte, en una investigación realizada por Restrepo et al. (2017), en Colombia, se encontró que la percepción de los docentes es que las instituciones no acompañan activa ni asertivamente los procesos de restablecimiento de los derechos del estudiantado. En este contexto, se evidencia la importancia de que los docentes desarrollen características personales que les ayuden a afrontar asertivamente las situaciones de violencia de su alumnado e incluso del contexto, siendo la resiliencia una capacidad importante para lograrlo.

En la institución 24 de mayo No. 2 cada docente está a cargo de 40 estudiantes, aproximadamente, por aula, lo que implica acciones como el aseguramiento del aprendizaje de cada uno de ellos, el control de disciplina, los trabajos administrativos, entre otras. Con relación al abordaje de casos de NNAVIF, los profesores aceptan que existe una dificultad en el seguimiento solo desde las herramientas de las políticas públicas o de los protocolos de las instituciones educativas.

El estudio realizado detectó que en el 71\% de los docentes existe una afectación emocional al momento de conocer un caso de NNAVIF, por lo que es necesario continuar trabajando en el fortalecimiento de sus capacidades personales y de bienestar emocional. No obstante, se evidencia que existe un nivel alto de resiliencia en los docentes, que les permite buscar diferentes tipos de estrategias y acciones para abordar, afrontar y acompañar a sus estudiantes.

Se recomienda que cada centro educativo cuente con más profesionales del área de psicología que trabajen en articulación con los centros de salud y justicia que abordan las situaciones de violencia intrafamiliar. Asimismo, es necesario contar con programas que permitan resguardar la salud mental y emocional de los docentes, que trabajen la prevención del agotamiento emocional y el burnout profesional. Además, se requiere mayor formación en herramientas psicoeducativas para el acompańamiento de los NNA que viven situaciones de violencia intrafamiliar. Se sugiere la creación de grupos de apoyo entre docentes, autoridades y estudiantes para fomentar prácticas positivas de erradicación de la violencia en todos sus ámbitos. Finalmente, se recomienda también realizar más investigaciones en torno al nivel de burnout y afectación emocional en los docentes que trabajan con casos de NNAVIF. 


\section{Referencias}

Acevedo, M. \& Mondragón, H. (2005). Resiliencia y escuela. Pensamiento Psicológico, 1(5), 21-35. http://www.redalyc.org/articulo.oa?id= 80100503

Díaz, C. \& Barra, E. (2017). Resiliencia y satisfacción laboral en profesores de colegios municipales y particulares subvencionados de la comuna de Machalí. Estudios Pedagógicos, 43(1), 75-86. https://scielo.conicyt.cl/scielo.php?script=sci_ abstract\&pid=S0718-07052017000100005\& $\operatorname{lng}=\mathrm{es} \& \mathrm{nrm}=\mathrm{iso}$

Folco, M. (2013). Estrategias docentes en la inclusión de niños y niñas en situación de vulnerabilidad. Revista internacional de educación para la justicia social, 2(2), 255-270. http://www.rinace. net/ riejs/numeros/vol2-num2/art12.pdf

Forján, R. \& Morelatto, G. (2018). Estudio comparativo de factores de resiliencia en docentes de contextos socialmente vulnerables. Psicogente, 21(40), 277-296. Doi: https://doi.org/10. $17081 /$ psico. 21.40 .3075

Franco, C. (2010). Intervención sobre los niveles de burnout y resiliencia en docentes de educación secundaria a través de un programa de conciencia plena (mindfulness). Complutense de Educación, 21(2), 271-288. https://revistas. ucm.es/index.php/RCED/article/view/RCED1010220271A

Gómez, A. \& Almeida, M. (2016). La resiliencia del docente como factor crucial para superar las adversidades en una sociedad de cambios. Tendencias pedagógicas, 28(1). 167-179. https:// revistas.uam.es/tendencias\%20pedagogicas/ article/view/3831

Instituto Nacional De Estadísticas Y Censos (INEC). (2018). Atlas de género. https://www.ecuadorencifras.gob.ec/documentos/web-inec/Bibliotecas/ Libros/Atlas_de_Genero_Final.pdf

Lagos, M. \& Ossa, C. (2010). Representaciones acerca de la resiliencia en educación según la opinión de los actores de la comunidad educativa. Horizontes Educacionales, 15(1), 37-52. http://www.redalyc. org/articulo.oa? id= 97916218004
Ley contra la violencia a la mujer y a la familia 2018, de 23 de enero, registro oficial del Estado, 175, de 5 de febrero de 2018. https://www.igualdadgenero.gob.ec/wp-content/uploads/2018/02/ REGISTRO-OFICIAL-LEY-ORGA\% CC\%81NICA-INTEGRAL-PARA-PREVENIR-Y-ERRADICAR-LA-VIOLENCIA-CONTRA-LAS-MUJERES.pdf

Ministerio de Educación (MINEDUC). (2017). Protocolos de actuación frente a situaciones de violencia detectadas o cometidas en el sistema educativo. https://educacion.gob.ec/rutas-y-protocolos/

Muñoz, V. \& Sotelo, F. (2005). Educar para la resiliencia: un cambio de mirada en la prevención de situaciones de riesgo social. Complutense de Educación, 16(1), 107-124. https://revistas.ucm. es/index.php/RCED/article/view/16914

Plan Nacional para la erradicación de la violencia de género hacia la niñez, adolescencia y mujeres. (2009). Material no publicado. https:// www 2 .unwomen.org/-/media/field $\% 20$ office $\% 20$ ecuador/documentos/publicaciones/2015/2015_017\%20mjdhc\%20folleto\% 20pnevg.pdf?la $=$ es\&vs=2307

Restrepo, Y. \& García, J. (2017). Rol del agente educativo en activación de rutas de atención de violencias sexuales en la infancia. Ciencia y Sociedad, 42(3), 29-49. Doi: https://www. redalyc.org/articulo.oa?id=87053126003

Saavedra, E., Arévalo, F., Gajardo, L. \& Toledo, L. (2010). Manual para intervención en resiliencia. Chile: CEANIM.

Saavedra, E. \& Villalta, M. (2008a). Escala de resiliencia SV-RES para jóvenes y adultos. CEANIM. https:// www.academia.edu/26343874/Saavedra_-Villalta_SV-RES60_ESCALA_DE_RESILIENCIA_SV-RES

Saavedra, E. \& Villalta, M. (2008b). Medición de las características resilientes, un estudio comparativo en personas entre 15 y 65 ańos. Liberabit, 14(14), 31-40. http://www.scielo.org.pe/scielo. php?pid=S1729-48272008000100005\&script= sci_abstract

Saltijeral, M. \& Ramos, L. (2015). Identificación de estresores laborales y burnout en docentes de una 
secundaria para trabajadores del Distrito Federal. Salud mental, 38(5), 361-369. http://www. scielo.org.mx/scielo.php?script=sci_arttext\&pid= S0185-33252015000500361

Uriarte, J. (2006). Construir la resiliencia en la escuela. Building resilience in school. Psicodidáctica, 11(1), 7-24. https://www.redalyc.org/pdf/175/ 17514747002.pdf
Vicente, I. \& Gabari, M. (2019). Liderazgo Pedagógico en Educación Secundaria: aportaciones desde la Evaluación de Burnout-Resiliencia en Docentes. International Journal of Educational Leadership and Management, 7(1), 104-134. Doi: http://dx. doi.org/10.17583/ijelm. 2019.3519 